\title{
Comparison Between Courses of Home and Inpatients Mechanical Ventilation in Patients with Muscular Dystrophy in Japan
}

\author{
Toshio Saito ${ }^{1}$ and Katsunori Tatara ${ }^{2}$ \\ ${ }^{1}$ Division of Neurology, National Hospital Organization Toneyama National Hospital \\ ${ }^{2}$ Division of Pediatrics, National Hospital Organization Tokushima National Hospital \\ Japan
}

\section{Introduction}

In Japan, 27 hospitals specialize in treatment of muscular dystrophy patients, including inpatient care, of which 26 belong to the National Hospital Organization, and the other is the National Center of Neurology and Psychiatry. Since 1999, Japanese muscular dystrophy research groups investigating nervous and mental disorder have been developing a database of cases treated at these 27 institutions. In that regard, we conducted a survey of inpatients and home-mechanical ventilation patients (HMV patients) with muscular dystrophy and other neuromuscular disorders based on data collected by the National Hospital Organization and National Center of Neurology and Psychiatry.

Herein, we examined data obtained in order to evaluate efficacy of mechanical ventilation therapy for HMV patients and mechanical ventilation-dependent inpatients (MV inpatients) with those wards.

\section{Subjects and methods}

The database includes numbers of inpatients, gender, age, diagnosis, respiratory condition, nutritional state, number of death cases, causes of death, and other relevant findings from data collected annually on October 1 every year since 1999. Additionally we collected the data of HMV patients from 27 institutes for this study.

By using the database and newly collected HMV data, we analyzed the courses of HMV patients group and those of MV inpatients of wards. We compared data of these two groups. Examination points are mechanical ventilation periods, outcome of these two groups, and caregiver for HMV patients.

\subsection{Objective diseases}

Objective diseases of this study were muscular dystrophy and spinal muscular atrophy, in particular Duchenne muscular dystrophy and myotonic dystrophy. Amyotrophic lateral sclerosis was not included. 


\subsection{Patients introduced HMV after 1999}

The data which we requested 27 institutes specializing muscular dystrophy care was as follows; the number of patients introduced HMV after 1999, diagnosis of disease, gender, age at being introduced HMV, type of mechanical ventilation, such as non-invasive positive pressure ventilation (NPPV) or tracheostomy intermittent ventilation (TIV), present status, death cause for death case, main caregiver, and so on.

\subsection{Patients introduced MV in muscular dystrophy wards after 1999}

We selected data of newly MV introduced inpatients after 1999 from the database of the muscular dystrophy wards.

\section{Results}

\subsection{Demographic features of HMV patients group and MV inpatients}

\subsubsection{HMV patients group}

HMV patients group included 434 patients from 14 institutes. Gender was male: 356, female: 78. The number of representative disease were as follows; 262 patients with Duchenne muscular dystrophy, 60 myotonic dystrophy, 17 Becker muscular dystrophy, 16 limb-girdle muscular dystrophy, 14 spinal muscular atrophy, and so on (Table 1-1).

\begin{tabular}{|c|c|c|c|c|}
\hline Diagnosis & HMV & Impatient & Total & Death cases \\
\hline BMD & 17 & 35 & 52 & 10 \\
\hline CMD & 12 & 6 & 18 & 2 \\
\hline DMD & 262 & 476 & 738 & 96 \\
\hline EDMD & 2 & 0 & 2 & \\
\hline FCMD & 16 & 43 & 59 & 13 \\
\hline FSHD & 6 & 33 & 39 & 7 \\
\hline LGMD & 16 & 42 & 58 & 12 \\
\hline MD & 60 & 222 & 282 & 62 \\
\hline MG & 1 & 0 & 1 & \\
\hline SMA & 13 & 19 & 32 & 6 \\
\hline SPMA & 0 & 11 & 11 & 4 \\
\hline UCMD & 9 & 1 & 10 & \\
\hline Mitochondrila disease & 0 & 5 & 5 & 1 \\
\hline Distal myopathy & 2 & 3 & 5 & 2 \\
\hline Congenital myopathy & 8 & 12 & 20 & \\
\hline Glycogen storage disease & 2 & 1 & 3 & \\
\hline Other myopathies & 3 & 1 & 4 & \\
\hline Other dystrophies & 4 & 5 & 9 & \\
\hline Unknown & 1 & 0 & 1 & \\
\hline total & 434 & 915 & 1349 & 215 \\
\hline
\end{tabular}

BMD, Becker muscular dystrophy; CMD, congenital muscular dystrophy; DMD, Duchenne muscular dystrophy; EDMD, Emery-Dreifuss muscular dystrophy; FCMD, Fukuyama congenital muscular dystrophy; FSHD, facio-scapulo-humeral muscular dystrophy; LGMD, limb-girdle muscular dystrophy; MD, Myotonic dystrophy; MG, myasthenia gravis; SMA, spinal muscular atrophy; SPMA, spinal progressive muscular atrophy, UCMD, Ullrich congenital muscular dystrophy

Table 1-1. Details of disease (HMV: from 14 institutes ) 


\subsubsection{MV inpatients group}

MV inpatients group included 915 inpatients. Gender was male: 718, female: 197. The number of representative disease were as follows; 476 Duchenne muscular dystrophy, 222 myotonic dystrophy, 35 Becker muscular dystrophy, 58 limb-girdle muscular dystrophy, 19 spinal muscular atrophy, and so on (Table 1-1).

\subsubsection{Mean age at starting mechanical ventilation and type of ventilation}

The range of mechanical ventilation introduction age for HMV patients was 6.3 72.8 years old (mean 25.9), and that of MV inpatients was 0.0 78.0 years old (mean 33.2). The number of NPPV introduction cases of HMV patients was 420, and that of MV inpatients was 517 (Table 1-2). Fifty of NPPV cases of HMV group were switched to tracheostomy during the course.

\begin{tabular}{lccc}
\hline & HMV & Inpatient & \\
\hline $\begin{array}{c}\text { Age at starting mechanical } \\
\text { ventilation (years old) }\end{array}$ & 25.9 & 33.2 & $\mathrm{p}<0.05$ \\
NPPV introduced case & 420 & 517 & \\
\hline
\end{tabular}

Table 1-2. Mean age at starting mechanical ventilation and type of ventilation

\subsection{Survival analysis of HMV patients group and MV inpatients}

We performed survival analysis of those two groups. The endpoint for HMV patients was death or transition to hospitalization, and that for MV inpatient was death. Kaplan-Meier analysis showed that 75\% life time of HMV patients was 1,689 days, while that of inpatients was 2,988 days (Log Rank (Mantel-Cox) p<0.01) (Fig. 1).

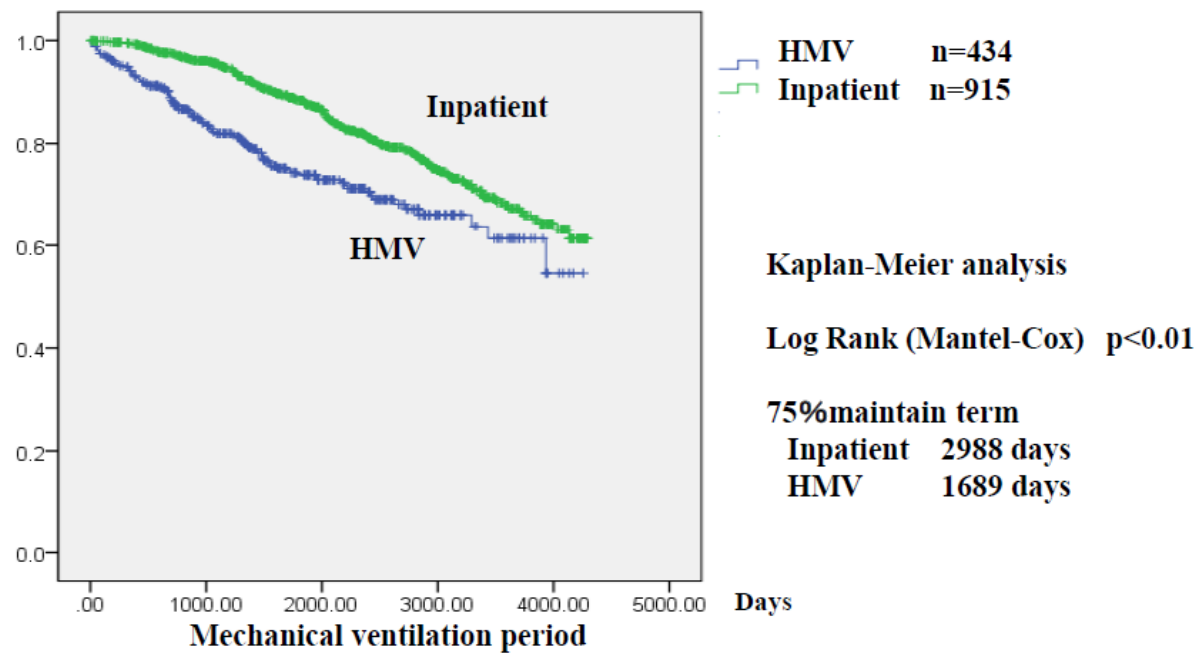

Fig. 1. Comparison between HMV Patients and Mechanical Ventilation Inpatients (Total) Endpoint for HMV patient: death or transition to hospitalization Endpoint for MV inpatient: death 


\subsection{Analysis data of Duchenne muscular dystrophy and myotonic dystrophy}

As the number of patients with Duchenne muscular dystrophy and myotonic dystrophy was great in these two groups, we analyzed the data of Duchenne muscular dystrophy and myotonic dystrophy separately.

\subsubsection{Mean age at starting mechanical ventilation and type of ventilation of patients with Duchenne muscular dystrophy}

Mean age at starting mechanical ventilation of Duchenne muscular dystrophy was 19.8 years old, ranged from 11.5 to 39.9 years old. While that of inpatient with Duchenne muscular dystrophy was 21.5 years old, ranged from 10.0 to 42.0 years old. There was no significant difference. The number of NPPV introduction cases of HMV patients with Duchenne muscular dystrophy was 220, and that of MV inpatients was 338 (Table 2).

\begin{tabular}{cccc}
\hline & HMV & Inpatient & \\
\hline $\begin{array}{c}\text { Age at starting mechanical } \\
\text { ventilation(years old) }\end{array}$ & 19.8 & 21.5 & NS \\
\hline NPPV & 220 & 338 & \\
TIV & 42 & 138 & \\
\hline total & 262 & 476 & \\
\hline \hline
\end{tabular}

Table 2. Mean age at starting mechanical ventilation and type of mechanical ventilation (Duchenne muscular dystrophy)

\subsubsection{Type of nutrition of patients with Duchenne muscular dystrophy}

The number of patients who required tube feeding, including a nasal or oral nutrition tube, and undergoing a percutaneous endoscopic gastrostomy (PEG) was apparently greater in MV inpatients group than HMV group (Table 3).

\begin{tabular}{ccc}
\hline & HMV & Inpatient \\
\hline Oral nutritional supply & 118 & 314 \\
PEG & 11 & 78 \\
Tube feeding & 3 & 71 \\
(per nasal or per oral) & 0 & 13 \\
Intravenous hyperamelitaion & 130 & 0 \\
unknown & 262 & 476 \\
\hline \hline total &
\end{tabular}

Table 3. Type of nutrition (Duchenne muscular dystrophy)

\subsubsection{Survival analysis of two Duchenne muscular dystrophy groups}

We performed survival analysis of those two Duchenne muscular dystrophy groups. The endpoint for HMV patients was death or transition to hospitalization, and that for MV inpatient was death. Kaplan-Meier analysis showed that $75 \%$ life time of HMV patients was 1,562 days, while that of inpatients was 3,739 days (Log Rank (Mantel-Cox) p<0.01) (Fig. 2). 


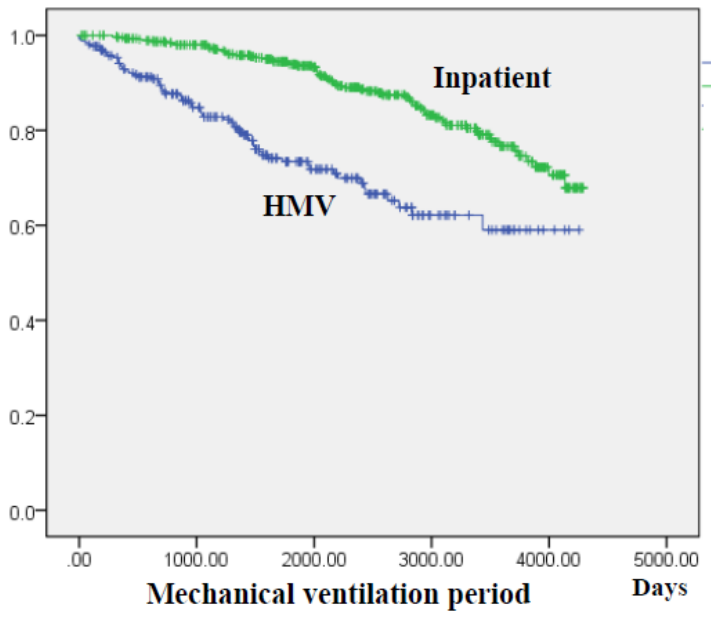

$$
\begin{array}{ll}
\text { HMV } & n=262 \\
\text { Inpatient } & \mathbf{n}=\mathbf{4 7 6}
\end{array}
$$

Kaplan-Meier analysis

Log Rank (Mantel-Cox) $\quad \mathbf{p}<0.01$

$75 \%$ maintain term

Inpatient 3739 days

HMV 1562 days

$50 \%$ maintain term

HMV 3475 days

Fig. 2. Comparison between HMV Patients and Mechanical Ventilation Inpatients

(Duchenne muscular dystrophy )

Endpoint for HMVpatient: death or transition to hospitalization

Endpoint for MV inpatient: death

\subsubsection{Mean age at starting mechanical ventilation and type of ventilation of patients with myotonic dystrophy}

Mean age at starting mechanical ventilation of myotonic dystrophy was 46.8 years old, ranged from 15.8 to 72.8 years old. While that of inpatient with myotonic dystrophy was 50.6 years old, ranged from 12.0 to 76.0 years old. There was no significant difference between two groups. The number of NPPV introduction cases of HMV patients with myotonic dystrophy was 55, and that of TIV was 5. The number of NPPV case was greater than TIV. While the number of NPPV introduction cases of MV patients with myotonic dystrophy was 108, and that of TIV was 114. In MV patients with myotonic dystrophy, the number and proportion of NPPV and TIV were almost equal (Table 4).

\begin{tabular}{cccc}
\hline & HMV & Inpatient & \\
\hline $\begin{array}{c}\text { Age at starting mechanical } \\
\text { ventilation (years old) }\end{array}$ & 46.8 & 50.6 & NS \\
\hline NPPV & 55 & 108 & \\
TIV & 5 & 114 & \\
\hline total & 60 & 222 & \\
\hline \hline
\end{tabular}

Table 4. Mean age at starting mechanical ventilation and type of mechanical ventilation (myotonic dystrophy )

\subsubsection{Type of nutrition of patients with myotonic dystrophy}

The trend of nutrition was similar to Duchenne muscular dystrophy. The number who required tube feeding in MV inpatients group was apparently greater than HMV group (Table 5). 


\begin{tabular}{ccc}
\hline & HMV & Inpatient \\
\hline Oral nutritional supply & 18 & 93 \\
PEG & 1 & 69 \\
Tube feeding & 0 & 50 \\
(per nasal or per oral) & 0 & 10 \\
Intravenous hyperamelitaion & 41 & 0 \\
\hline unknown & 60 & 222 \\
\hline \hline total &
\end{tabular}

Table 5. Type of nutrition (myotonic dystrophy)

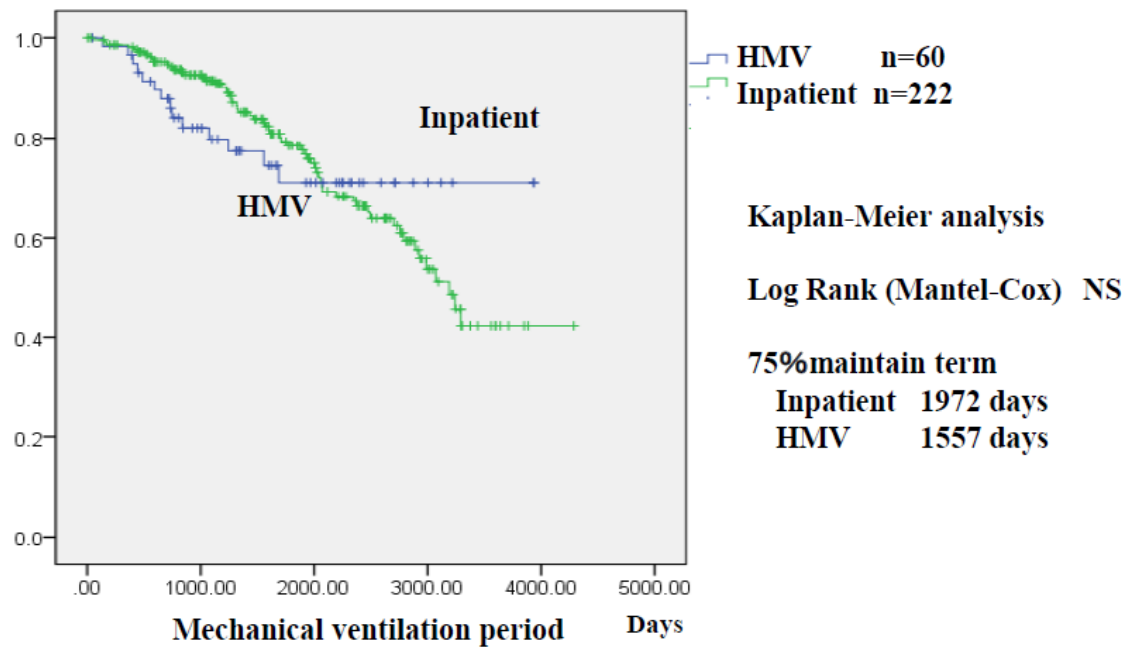

Fig. 3. Comparison between HMV Patients and Mechanical Ventilation Inpatients (myotonic dystrophy)

Endpoint for HMVpatient: death or transition to hospitalization

Endpoint for MV inpatient: death

\subsubsection{Survival analysis of two myotonic dystrophy groups}

Similarly, we performed survival analysis of those two myotonic dystrophy groups. The endpoint for HMV patients was death or transition to hospitalization, and that for MV inpatient was death. Kaplan-Meier analysis showed that $75 \%$ life time of HMV patients was 1,557 days, while that of inpatients was 1,972 days. There was no significance (Fig. 3).

\subsection{Death case}

The total number of death cases was 215 (Table 1-1). As the number of death case of Duchenne muscular dystrophy and myotonic dystrophy was the majority, we analyzed the data of Duchenne muscular dystrophy and myotonic dystrophy separately.

\subsubsection{Cause of death of Duchenne muscular dystrophy}

The number of death cases of HMV patients was 29, and that of MV inpatients was 67. 
The most frequent cause of death was heart related disorders, such as heart failure and arrhythmia, accounting for 16/29 for HMV group and 33/67 for MV inpatient group. Frequency was not significantly different between two groups. In MV inpatient group, respiratory related disorders, such as respiratory failure and respiratory infection, accounted for 23/67. HMV group included more sudden death cases than MV inpatient, and had an accidental case (Table 6).

\begin{tabular}{cccc}
\hline & HMV & Inpatient & Total \\
\hline Heart failure & 13 & 27 & 40 \\
Arrhythmia & 3 & 6 & 9 \\
Respiratory failure & 2 & 11 & 13 \\
Respiratory infection & 1 & 12 & 13 \\
Tracheal bleeding & 1 & 0 & 1 \\
Pneumothorax & 0 & 1 & 1 \\
Renal failure & 0 & 1 & 1 \\
Infectious disease & 2 & 0 & 2 \\
Malignancy & 1 & 0 & 1 \\
Ileus & 1 & 0 & 1 \\
Sudden death & 4 & 2 & 6 \\
Power supply accident & 1 & 0 & 1 \\
Others & 0 & 7 & 7 \\
\hline \hline total & 29 & 67 & 96 \\
\hline
\end{tabular}

Table 6. Cause of death (Duchenne muscular dystrophy )

\subsubsection{Cause of death of myotonic dystrophy}

The majority of death case of myotonic dystrophy was reported from MV inpatient group. The most frequent cause was respiratory related disorders, such as respiratory tract infection and respiratory failure, which accounted for 29/56. Sudden death case was conspicuous in HMV group, accounting for 3/6(Table 7).

\begin{tabular}{cccc}
\hline & HMV & Inpatient & Total \\
\hline Respiratory & 1 & 19 & $\mathbf{2 0}$ \\
infection & 2 & 10 & 12 \\
Respiratory failure & 2 & 8 & 8 \\
Heart failure & 0 & 1 & 1 \\
DIC & 0 & 2 & 2 \\
MOF & 0 & 2 & 2 \\
Cholangitis & 0 & 1 & 1 \\
Ileus & 0 & 1 & 1 \\
Choking & 0 & 2 & 2 \\
Malignancy & 0 & 1 & 1 \\
Intestinal bleeding & 0 & 1 & 1 \\
Hepatic failure & 0 & 2 & 5 \\
Sudden death & 3 & 6 & 6 \\
Others & 0 & $\mathbf{5 6}$ & $\mathbf{6 2}$ \\
\hline \hline total & $\mathbf{6}$ & &
\end{tabular}

Table 7. Cause of death (myotonic dystrophy) 


\subsection{Outcome of HMV patients and MV inpatients with Duchenne muscular dystrophy and myotonic dystrophy}

One hundred ninty four cases with Duchenne muscular dystrophy among 262 cases continued HMV, while 46 cases with myotonic dystrophy among 60 cases continued HMV (Table 8).

Twenty two cases with Duchenne muscular dystrophy were switched to hospitalization.

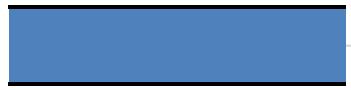

Continuing HMV

Continuing hospitalization

Death

Transition to hospitalization

Introduction to other institution

Withdrawing MV

\begin{tabular}{c}
$\begin{array}{c}\text { Unknown } \\
\text { Others }\end{array}$ \\
\hline total
\end{tabular}

\begin{tabular}{cr}
\hline \multicolumn{2}{c}{ DMD } \\
HMV Inpatient \\
\hline
\end{tabular}

194

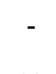

29

22

407

67

3

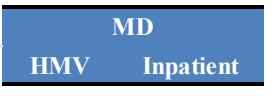

46

$-\quad 151$

$6 \quad 56$

3

10

- $\quad-$

$7 \quad 2$

$\begin{array}{llll}- & - & 2 & 15\end{array}$

$262 \quad 476$

$60 \quad 222$

Table 8. Outcome (Duchenne muscular dystrophy and myotonic dystrophy )

\subsection{Caregivers for HMV patients}

Caregivers for most of HMV patients with Duchenne muscular dystrophy were patients' families (Table 9). In particular, patients' mothers were playing important role in continuing HMV. Similarly, caregivers for HMV patients with myotonic dystrophy were patients' families. On reflecting their age, some caregivers were patient' spouse (Table 9).

\begin{tabular}{ccc}
\hline Caregiver & DMD & MID \\
\hline Mother & 94 & 6 \\
Parents & 22 & \\
Father & 3 & 2 \\
Family & 3 & \\
Mother/sibling & 1 & \\
Mother/uncle & 1 & \\
Mother/grandmother & 1 & 2 \\
Grandmother & 1 & 1 \\
Husband & & 1 \\
Wife & & 1 \\
Sister & & 2 \\
daughter & & 2 \\
foundation & & $(17 / 60)$ \\
Helper & 2 &
\end{tabular}

Table 9. HMV-continuing cases main caregiver (Duchenne muscular dystrophy and myotonic dystrophy) 


\subsection{Summary of results of Duchenne muscular dystrophy and myotonic dystrophy}

Proportion of TIV was higher in MV inpatients group than HMV group. And proportion of tube feeding was also higher in MV inpatients group than HMV group. Namely, respiratory condition and nutritional status were more severe in MV inpatients group than HMV group (both Duchenne muscular dystrophy and myotonic dystrophy).

In Survival analysis, outcome of patients with Duchenne muscular dystrophy of MV inpatients group was better than HMV group. Meanwhile in that of myotonic dystrophy, HMV group was better than inpatient group.

In MV inpatient group of Duchenne muscular dystrophy, respiratory related death was remarkable.

In HMV group, some sudden death cases and accidental death case were conspicuous.

Caregivers of HMV group were constructed by patients' families, centrally mother.

\section{Conclusion}

Approximate 2500 beds for patients with muscular dystrophy and related disorders are now provided among 27 institutions in Japan. In accordance with progress in therapeutic strategies for respiratory failure (American Thoracic Society Documents, 2004) and heart failure (Ishikawa, 1999; Matsumura, 2010), the life span of patients with muscular dystrophy prolonged (Bushby 2010a, b). Now, most inpatients admitted to muscular dystrophy wards have severe general conditions and many are assisted by mechanical ventilation (Tatara, 2006; 2008), which is accordance with our data of MV patients in this study.

In recent two decades, social welfare systems and home medical care systems in Japan have been changing gradually. HMV has been penetrating into home medical care (Joseph, 2007). The number of HMV patients has been increasing (Tatara, 2006). Stable mechanical ventilated patients have been getting back home.

Our study demonstrated that the course of HMV patients was fairly good, although there was difference between Duchenne muscular dystrophy and myotonic dystrophy in long term outcome. However, the support system for patients and caregivers is not perfect. Our study also showed that burden of caregivers was supposed to be severe. The system for patients and caregivers should be adjusted (Dybwik, 2011). And safety net systems also should be adjusted to avoid accidental event leading to patient's death.

The muscular dystrophy wards may be requested to offer the circumstances for those who have difficulties in continuing HMV. There is necessarily needs for hospitalization of HMV patients (Windisch, 2008).

Study limitation: This study has limitation on bias of collecting patients' information. Specifically, information of HMV patients were reported from 14 institutes among 27 institutes, and MV inpatient information is the result of extraction from muscular dystrophy wards database. Extracted data from database has some ambiguous points in connection with obscure time-sequential analysis. 
On analysis of institutes-restricted HMV patients group and MV inpatients group, differences in regard to therapeutic conditions among institutions may be problem.

\section{Acknowledgments}

This study was supported by a Research Grant for Nervous and Mental Disorders from the Ministry of Health, Labour and Welfare of Japan.

We are grateful to the members of the FUKUNAGA (1999-2005) and SHINNO (2006-2011) muscular dystrophy research groups of the National Hospital Organization for the data collection.

Institutions specializing in muscular dystrophy treatment in Japan (Fig.4)

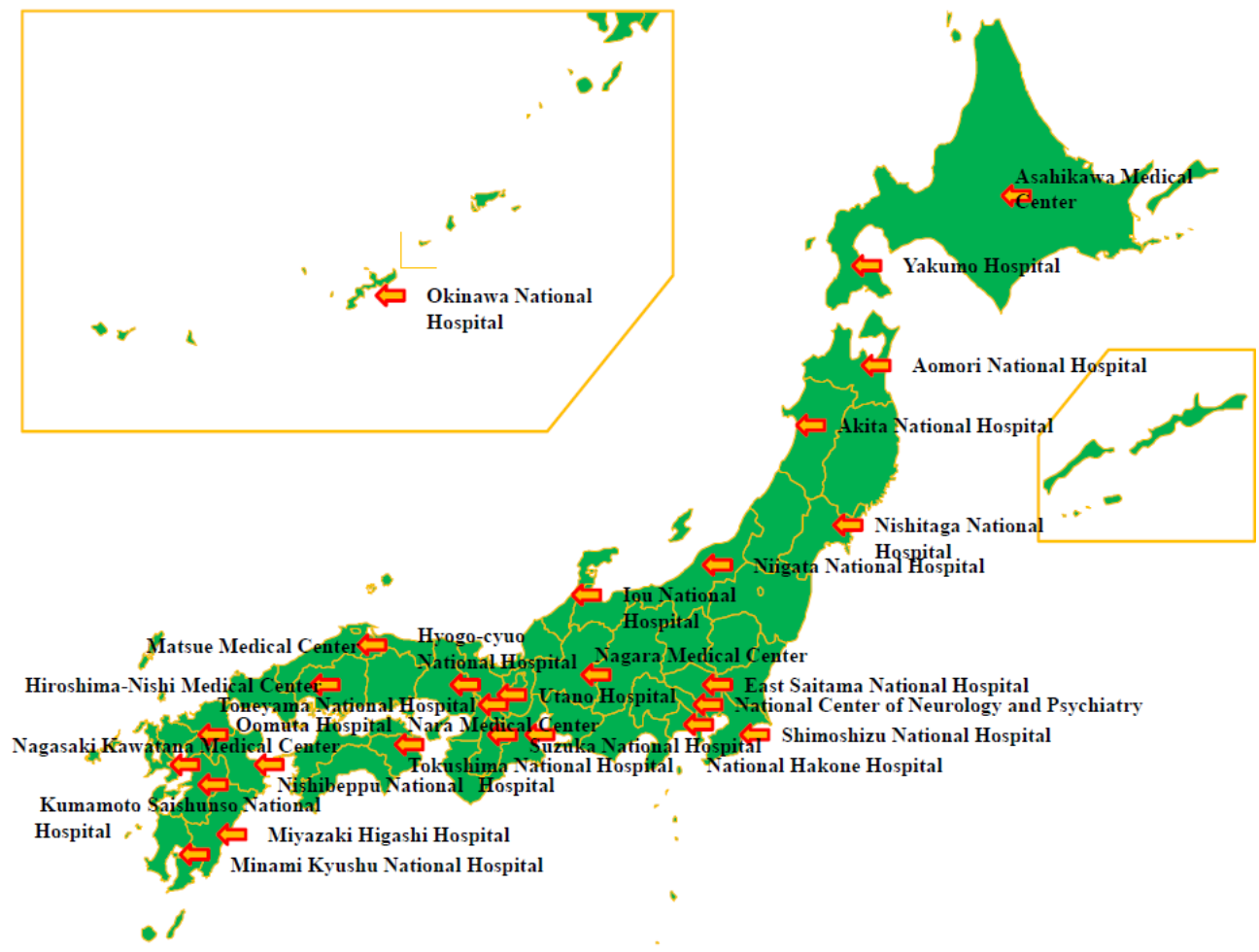

Fig. 4. Institutions specializing in muscular dystrophy treatment in Japan 


\section{National Hospital Organization:}

Asahikawa Medical Center, Yakumo Hospital, Aomori National Hospital,

Akita National Hospital, Nishitaga National Hospital, East Saitama National Hospital,

Shimoshizu National Hospital, National Hakone Hospital, Niigata National Hospital,

Iou National Hospital, Nagara Medical Center, Suzuka National Hospital,

Nara Medical Center, Utano Hospital, Toneyama National Hospital,

Hyogo-cyuo National Hospital, Hiroshima-Nishi Medical Center, Matsue Medical Center,

Tokushima National Hospital, Oomuta Hospital, Nagasaki Kawatana Medical Center,

Kumamoto Saishunso National Hospital, Nishibeppu National Hospital,

Miyazaki Higashi Hospital, Minami Kyushu National Hospital, Okinawa National Hospital

\section{National Center of Neurology and Psychiatry}

\section{References}

American Thoracic Society Documents (2004). Respiratory Care of the Patient with Duchenne Muscular Dystrophy. ATS Consensus Statement American Journal of Respiratory and Critical Care Medicine, Vol 170, pp 456-465.

Bushby K, Finkel R, Birnkrant DJ, Case LE, Clemens PR, Cripe L, Kaul A, Kinnett K, McDonald C, Pandya S, Poysky J, Shapiro F, Tomezsko J, Constantin C, for the DMD care considerations working group (2010a). Diagnosis and management of Duchenne muscular dystrophy, part 1: diagnosis, and pharmacological and psychosocial management. The Lancet Neurology, Vol.9, pp 77-93.

Bushby K, Finkel R, Birnkrant DJ, Case LE, Clemens PR, Cripe L, Kaul A, Kinnett K, McDonald C, Pandya S, Poysky J, Shapiro F, Tomezsko J, Constantin C, for the DMD care considerations working group (2010b). Diagnosis and management of Duchenne muscular dystrophy, part 2: implementation of multidisciplinary care. The Lancet Neurology, Vol.9, pp 177-189.

Dybwik.K, Nielsen.E.W,Brinchmann. B. S (2011).Home mechanical ventilation and specialized health care in the community: Between a rock and a hard place. BMC Health Services Research, Vol. 11, pp 115-123.

Ishikawa Y, Bach JR, Minami R (1999). Cardioprotection for Duchenne's muscular dystrophy.American Heart Journal Vol.137, pp 895-902.

Joseph S. L, Peter C. G (2007). Current Issues in Home Mechanical Ventilation. Chest, Vol. 132, pp 671-676.

Matsumura T, Tamura T, Kuru S, Kikuchi Y, Kawai M (2010) · Carvedilol can prevent cardiac events in Duchenne muscular dystrophy. Internal Medicine Vol. 49, pp 13571363.

Tatara K, Fukunaga H, Kawai M (2006). Clinical survey of muscular dystrophyin hospitals of National Hospital Organization. IRYO, Vol. 60, pp 112-118. 
Tatara K, Shinno S (2008). Management of mechanical ventilation and prognosis in Duchenne muscular dystrophy. IRYO, Vol. 62, pp 566-571.

Windisch W; Quality of life in home mechanical ventilation study group (2008). Impact of home mechanical ventilation on health-related quality of life. Eur Respir J. Vol. 32, pp 1328-1336. 
(C) 2012 The Author(s). Licensee IntechOpen. This is an open access article distributed under the terms of the Creative Commons Attribution 3.0 License, which permits unrestricted use, distribution, and reproduction in any medium, provided the original work is properly cited. 